\title{
Coronary Pan-Ischemia as a First Sign of a Fulminant Host-Versus-Graft Reaction Eight Years After Orthotopic Heart Transplantation
}

\author{
Juergen KAMmLeR, ${ }^{1}$ MD, Hermann Blessberger, ${ }^{1}$ MD, Alexander Kypta, ${ }^{1}$ MD, \\ Michael LichtenAuer, ${ }^{2}$ MD, Alexander NAhler, ${ }^{1}$ MD, Thomas LAmbert, ${ }^{1}$ MD, \\ Klaus Aumayr, ${ }^{3} \mathrm{MD}$, Andreas Zuckermann, ${ }^{4} \mathrm{MD}$, and Clemens Steinwender, ${ }^{1} \mathrm{MD}$
}

\begin{abstract}
SUMMARY
Acute graft rejection in patients after heart transplantation can cause arrhythmias and acute angina pectoris with electrocardiographic ST-segment elevation. We report a case of a 53-year old female patient who had undergone cardiac transplantation 8 years previously. She developed bradycardia with co-existent ST-segment elevation caused by a histologically proven acute graft rejection. After administration of methylprednisolone and immune absorption leading to initial clinical improvement, the patient died unexpectedly. The reasons remain unclear, but a degeneration of the conduction system as well as impaired blood flow in the right coronary caused by cellular and humoral rejection most likely have both contributed. (Int Heart J 2015; 56: 679-681)
\end{abstract}

Key words: Graft rejection, ST-segment elevation, Heart failure

$\mathrm{H}$ ost-versus-graft reaction is the most important complication in patients undergoing heart transplantation in long-term follow-up. The impact on myocardial dysfunction is well known and described. We present a case in which inflammatory damage of the conduction system may have led to fatal outcome in this very patient.

\section{Case Report}

We report the case of a 53-year-old female patient who had undergone 3 aortic homograft replacements between 1994 and 1995 due to recurrent bacterial and fungoid endocarditis. Due to coronary artery disease present at the time of the third operation, aortocoronary bypass surgery was performed in addition to the aortic homograft implantation with saphenous vein grafts to the left anterior descending artery and circumflex artery. The clinical course appeared uneventful during the following years.

In 2002, the patient was admitted due to biventricular heart failure NYHA class III-IV, based on a valvular cardiomyopathy with a significantly reduced left ventricular ejection fraction caused by severe degeneration of the aortic homograft prosthesis with significant insufficiency of the valve.

Coronary angiography was performed and neither pathological findings in the bypass grafts nor progression of coronary artery disease in the native vessels was observed.
Optimal medical treatment did not result in an improvement of exercise tolerance and left ventricular ejection fraction.

Due to deterioration of her condition she underwent implantation of a Thoratec Assist Device 2/2003 as a bridge to candidacy.

Consequently, the patient was listed for heart transplantation. In May 2003, orthotopic heart transplantation was performed at the Medical University of Vienna.

During the following years when on a triple immunosuppressive regimen (calcineurin inhibitors, mycophenolatemofetil and steroids), she reported a good quality of life with satisfying long-term follow-up parameters. ${ }^{1)}$

In 2011, the patient was admitted to our intensive care unit due to an episode of chest pain, that lasted for 15 minutes. The first electrocardiogram, without clinical symptoms, showed normal ST-segments. One hour after admission, another episode of chest pain occurred, accompanied by substantial ST-segment elevation in all leads, which was interpreted as cardiac pan-ischemia (Supplemental Figure 1A, B).

Urgent coronary angiography was performed after administration of a bodyweight adapted bolus of abciximab $(0.25$ $\mathrm{mg} / \mathrm{kg}$ ). It revealed a normal left ventricular ejection fraction and no relevant graft sclerosis ${ }^{2,3)}$ with no significant coronary stenosis but an impaired coronary blood flow (TIMI grade IIIII) of all native vessels, especially the right coronary artery.

In addition, we found a moderate to severe ischemic mitral regurgitation, mainly caused by hypokinesis of the postero-

From the ${ }^{1}$ 1st Medical Department - Cardiology, Linz General Hospital, Johannes Kepler University Linz School of Medicine, Linz, ${ }^{2}$ Department of Internal Medicine II, Paracelsus Medical University Salzburg, Salzburg, and Departments of ${ }^{3}$ Pathology and ${ }^{4}$ Cardiac Surgery, Medical University of Vienna, Vienna, Austria.

Address for correspondence: Juergen Kammler, MD, Linz General Hospital, 1st Medical Department - Cardiology, Johannes Kepler University School of Medicine, Krankenhausstrasse 9, A-4021 Linz, Austria. E-mail: juergen.kammler@akh.linz.at

Received for publication May 27, 2015. Revised and accepted June 2, 2015.

Released in advance online on J-STAGE November 4, 2015.

All rights reserved by the International Heart Journal Association. 
basal segments of left ventricle (Supplemental Figure 2).

We hypothesized that an acute host-versus-graft reaction could be the underlying cause of the symptoms, and administered 2 grams of methylprednisolone intravenously within 48 hours. In addition to immunosuppressive therapy, we administered bivalirudin intravenously (body weight adapted bolus of $0.75 \mathrm{mg} / \mathrm{kg}$, followed by an infusion of $1.75 \mathrm{mg} / \mathrm{kg} /$ hour), as we considered diffuse micro-embolization to the coronary vessels leading to the impaired TIMI flow as a differential diagnosis.

The patient was free from chest pain after this treatment. Electrocardiographic monitoring did not reveal further episodes of cardiac ischemia or arrhythmias.

The following day, the patient was transferred to the transplant center in Vienna. Endomyocardial biopsy, performed on the third day after symptom onset, revealed moderate graft rejection (3A/2R pAMR). ${ }^{4}$ The histological pattern showed signs of cellular as well as humoral rejection with pericapillary deposits of C3d and C4d (Supplemental Figure 3A, B).

In addition, donor specific antibodies were detected. Another 1.5 grams of methylprednisolone was administered within 3 days and immune absorption was performed. This led to a significant reduction of antibodies and the patient's condition improved over the next few days, with the exception of one episode of short asymptomatic bradycardia. Several electrocardiograms showed no signs of conduction delay, such as prolongation of the PR-interval or bundle branch block.

Control endomyocardial biopsy after one week showed an improvement of all aspects of the graft rejection.

Serial echocardiographic assessments showed persistent mitral regurgitation. This regurgitation was interpreted as ischemic due to suspected micro-embolization to the periphery of the right coronary artery.

As mitral regurgitation was interpreted as being clinically relevant, the patient was scheduled for elective mitral valve repair. The day before the planned surgery, she developed bradycardia which made cardiopulmonary resuscitation necessary. However, all efforts were unsuccessful and she died 34 days after the first symptoms.

Histological examination of the heart showed an alleviated lymphocytic host-versus-graft reaction, but no further specific changes which could help to clarify the cause of death.

\section{Discussion}

The cause of death could not be definitively identified in this patient. However, there are some important issues to be pointed out.

Acute pump failure due to a severe prolonged episode of graft rejection $^{5)}$ seems unlikely to have caused her death as she was free from symptoms over several days and showed no signs of heart failure on the day of death. The histological findings postmortem are in line with these assumptions.

An episode of severe bradyarrhythmia due to transient conduction delay with total atrioventricular block resulting in asystole might be another explanation. Several reports suspect transplant rejection to be responsible for arrythmogenic events in patients after heart transplantation, while in other reports autopsy results revealed marked involvement of the conduction system in acute graft rejections. ${ }^{6-10)}$ In addition, electrophysio- logical studies in animal models show that the most sensitive parameter of acute rejection is a prolonged atrioventricular conduction time. $^{11)}$

In our case, this mechanism might have caused inflammatory damage to the conduction system, especially the atrioventricular node, leading to the one documented and then the suspected fatal bradyarrhythmogenic event.

In contrast, it is also possible that micro-embolic events of the right coronary artery caused ischemia of the atrioventricular nodal artery with intermittent complete atrioventricular block and consecutively asystole as it is very well described in patients with acute coronary syndrome. ${ }^{12)}$

In conclusion, we think that patients with graft rejection after heart transplantation should be assessed and monitored for signs of cardiac conduction disturbances. Intermittent or permanent pacemaker therapy may then be indicated in order to prevent sudden cardiac death due to atrioventricular block and asystole.

\section{Disclosure}

There are no commercial associations that might pose a conflict of interest. This statement holds true for all authors.

\section{REFERENCES}

1. Oda N, Kato TS, Komamura K, et al. Clinical course and outcome of heart transplant recipients. Int Heart J 2010; 51: 264-71.

2. Ishida J, Kinugawa K, Shiga T, et al. Rapidly progressive cardiac allograft vasculopathy in early onset regressed with everolimus treatment in an adult cardiac recipient. Int Heart J 2012; 53: 38890.

3. Imamura $\mathrm{T}$, Kinugawa $\mathrm{K}$, Murasawa $\mathrm{T}$, et al. Cardiac allograft vasculopathy can be distinguished from donor-transmitted coronary atherosclerosis by optical coherence tomography imaging in a heart transplantation recipient. Int Heart J 2014; 55: 178-80.

4. Stewart S, Winters GL, Fishbein MC, et al. Revision of the 1990 working formulation for the standardization of nomenclature in the diagnosis of heart rejection. J Heart Lung Transplant 2005; 24: 1710-20.

5. Imamura T, Kinugawa $\mathrm{K}$, Kato N, et al. Successful treatment of hemodynamic compromise caused by antibody-mediated and cellular rejection in a recipient 12 years after heart transplantation. Int Heart J 2013; 54: 328-31.

6. Schroeder JS, Berke DK, Graham AF, Rider AK, Harrison DC. Arrhythmias after cardiac transplantation. Am J Cardiol 1974; 33 : 604-7.

7. Park JK, Hsu DT, Hordof AJ, Addonizio LJ. Arrhythmias in pediatric heart transplant recipients: prevalence and association with death, coronary artery disease, and rejection. J Heart Lung Transplant 1993; 12: 956-64.

8. Blanche C, Czer LS, Trento A, et al. Bradyarrhythmias requiring pacemaker implantation after orthotopic heart transplantation: association with rejection. J Heart Lung Transplant 1992; 11: 44652.

9. Jacquet L, Ziady G, Stein K, et al. Cardiac rhythm disturbances early after orthotopic heart transplantation: prevalence and clinical importance of the observed abnormalities. J Am Coll Cardiol 1990; 16: 832-7.

10. Knight CS, Tallaj JA, Rayburn BK, et al. Bradykardia and synkope as a presentation of cardiac allograft rejection involving the conduction system. Cardiovasc Pathol 2010; 19: 117-20.

11. Wnuk-Wojnar AM, Zembala M, Religa Z, et al. Electrophysiologic properties of transplanted human heart with and without rejec- 
tion. J Heart Lung Transplant 1992; 11: 435-41.

12. Braat SH, de Zwaan C, Brugada P, Coenegracht JM, Wellens HJ

Right ventricular involvement with acute inferior wall myocardial infarction identifies high risk of developing atrioventricular nodal conduction disturbances. Am Heart J 1984; 107: 1183-7.

\section{SuPPlemental Files}

Supplemental Figure 1, 2, 3

Please find supplemental file;

https://www.jstage.jst.go.jp/article/ihj/56/6/56_15-204/_article/supplement 